Do people really prefer verbal probabilities?

Marie Juanchich and Miroslav Sirota

University of Essex, Department of Psychology

Corresponding author: Marie Juanchich. Address: Department of Psychology, University of Essex, Wivenhoe Park, CO4 3SQ, Colchester, UK. Email: m.juanchich@essex.ac.uk

Acknowledgements: We thank all our coders, Dawn Liu, Alistair Thorpe, Anca Pop and Amalia Cerb 


\begin{abstract}
When people communicate uncertainty, do they prefer to use words (e.g., "a chance", "possible") or numbers (e.g., "20\%”, "a 1 in 2 chance")? To answer this question, past research drew from a range of methodologies, yet failed to provide a clear-cut answer. Building on a review of existing methodologies, theoretical accounts and empirical findings, we tested the hypothesis that the preference for a particular format is driven by the variant of uncertainty that people experience. We expected that epistemic uncertainty would be more often communicated in words, whereas distributional uncertainty would be more often communicated in numbers; for the dispositional uncertainty, we expected that an individual's disposition would be more often communicated in words, whereas dispositions from the world would be more often communicated numerically. In three experiments (one oral, two written), participants communicated their uncertainty regarding two outcomes per variants of uncertainty: epistemic, dispositional and distributional. Overall, participants communicated their uncertainty more often in words, but this preference depended on the variants of uncertainty. Participants conveyed their epistemic and dispositional uncertainties more often in words and their distributional uncertainty in numbers (Experiments 1 and 2) but this effect was greatly reduced when the precision of uncertainty was held constant (Experiment 3), pointing out the key role of uncertainty vagueness. We have reviewed the implications of our findings for the existing accounts of format preferences.
\end{abstract}

Keywords: uncertainty communication; format; verbal; numerical; variants of uncertainty 


\title{
Do people really prefer verbal probabilities?
}

\author{
"I don't know. It would probably be whether it would be likely \\ or unlikely. So it wouldn't be a percentage, it would just be a \\ verbal likely or unlikely."
}

The above example is a quote from one of the participants in our study. It clearly shows that when we communicate our uncertainty, we face a choice: whether or not we should express it in words (e.g., "there is a chance", "it is likely") or numbers (e.g., "a 20\% chance", "a 1 in 4 chance") and that in some cases this choice is not straightforward. In most of the past work on the subject, it has been claimed that naïve participants prefer to use verbal uncertainty phrases over numerical ones (Erev \& Cohen, 1990; Ho, Budescu, Dhami, \& Mandel, 2015; Honda \& Yamagishi, 2009; Juanchich, Sirota, \& Butler, 2012; Mullet \& Rivet, 1991; Reagan, Mosteller, \& Youtz, 1989; Renooij \& Witteman, 1999). This verbal uncertainty preference is also believed to be true for experts such as doctors (Mazur \& Hickam, 1991) or intelligence analysts (Dhami, Mandel, Mellers, \& Tetlock, 2015). The argument that people prefer verbal over numerical uncertainty phrases has implications for practice too, as it has been used (among others) to support prescriptive verbal risk communication guidelines in a range of applied settings (Budescu \& Wallsten, 1995; applied to climate change: Ho et al., 2015; applied to medicine: Renooij \& Witteman, 1999). However, the actual evidence of the preference for verbal uncertainty phrases is limited or even sometimes contradictory. Mirroring the empirical research, the existing theoretical accounts of the format preference have yet to account for the diversity of empirical findings to capture the mechanisms underpinning this preference. In this introduction, we review the literature regarding the uncertainty format that speakers prefer to use. We review evidence elicited by different methodologies, outline key theories explaining people's format 
preference and formulate an integrative theoretical account that is then tested in three experiments.

\section{Uncertainty communication format preference: Review of evidence}

Among the various uncertainty quantifiers, there are two main communication formats: numerical and verbal (see examples A and B).

A: There is a good chance that the sea will rise by 2 metres.

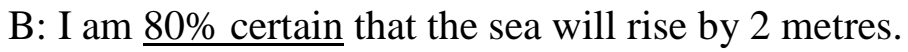

Both formats cover a range of different quantifiers. These include verbal probabilities, verbal frequencies or epistemic verbs for verbal uncertainty quantifiers, whereas ratios, odds, intervals and single numerical percentages are used for numerical quantifiers. Verbal formats are inherently vague, whereas numerical formats can range from very precise (e.g., single point estimates $-20 \%$ ) to very vague (e.g., ranges - from around $10 \%$ to $80 \%$ likely). In the context of assessing the format that people prefer to use to communicate their uncertainty, the most commonly studied verbal expressions of uncertainty are verbal probabilities (example A) and the most common numerical format studied is the point numerical percentage estimate (example B) (Brun \& Teigen, 1988; Erev \& Cohen, 1990; Juanchich \& Sirota, In press; Xu, Ye, \& Li, 2009). These two quantifiers are often used to operationalise their respective formats, pitting, for example, the preference for single estimate percentages against the preference for verbal probabilities. The belief that people prefer verbal over numerical uncertainty phrases is based on evidence stemming from three main paradigms: natural speech corpus studies, selection tasks and production tasks.

Evidence from natural speech corpus studies. Corpus studies have identified the fact that verbal uncertainty phrases are diverse and commonly occur in natural speech (i.e., speech 
that is not utilised for research purposes, such as the speech of TV presenters, text in medical reports, blogs). For example, a range of verbal probabilities were found in TV news (Brun \& Teigen, 1988), medical reports (Clark, 1990), medical litigation (Merz, Druzdzel, \& Mazur, 1991), police profiling investigation reports (Almond, Alison, \& Porter, 2007; Collins \& Alison, 2002) and in phytosanitary risk assessment reports (MacLeod \& Pietravalle, 2017). Research focusing exclusively on numerical probabilities dovetails this trend. For example, a study showed that family physicians only provided a numerical estimate of risk in $11 \%$ of 70 clinical consultations regarding patients' cardiovascular risk. This indicates that family physicians favoured a verbal form of uncertainty communication (Neuner-Jehle, Senn, Wegwarth, Rosemann, \& Steurer, 2011).

This tendency to use verbal probabilities is sometimes channelled and reinforced by the official recommendations of leading organisations to use a specific uncertainty lexicon to communicate a range of risks such as the side effects of drugs (European Commission, 1998), climate change (Intergovernmental Panel on Climate Change, 2007, 2013), intelligence services (Homeland Security Risk Steering Committee, 2008; Ministry of Defence, 2011) and food safety (European Food Safety Authority, 2017). Other organisations use verbal probabilities to set critical decision-making standards. For example, the International Accounting Standards Board requires that accountants recognise revenue when economic benefits are "probable" (International Accounting Standard Committee, 1998), and most justice systems have a set standard of proof which leads to guilty verdicts when jurors believe, "beyond reasonable doubt", that the suspect is guilty.

The fact that verbal uncertainties are diverse and common implies that they are a useful part of people's vocabulary. This finding has sometimes been stretched to mean that verbal uncertainty is the most common way to express uncertainty. However, the high frequency of 
verbal uncertainty does not imply that verbal uncertainty is preferred over numerical uncertainty. In other words, common does not necessarily mean preferred. For instance, if quantifiers, in general, are commonly used in human language, then verbal as well as numerical probabilities might be frequent. The corpus studies focusing on the communication of uncertainty often focus on the frequency of use of either verbal or numerical uncertainty, but not both, preventing us from drawing a conclusion regarding the preference for a format relative to the other.

The strengths of corpus studies - "real" use of language, with a large amount of data readily available from a diverse population and on a wide range of topics - can also be seen as weaknesses. The text is produced in an uncontrolled environment and therefore can only have a descriptive value. One can describe the condition in which the sentences were uttered (e.g., in a right wing newspaper) and can even compare the format preference according to some contextual aspect (e.g., finance magazines use more numerical uncertainty phrases than philosophy magazines). However, one cannot establish a causal relationship between the context and the format used (e.g., we cannot conclude that talking about financial outcomes increases the use of numerical uncertainty phrases). The methodologies below allow to control for the context and even to manipulate it to identify the circumstances in which people prefer (or do not prefer) verbal uncertainty phrases.

Evidence from selection tasks. Selection tasks are also sometimes called forced choice tasks because participants are given a limited number of options from which to choose. Findings from selection tasks tend to indicate that people prefer verbal phrases to numerical ones; however, variants of the task - format selection and uncertainty phrase selection - provide less consistent results. 
In format selection tasks, participants select which format they prefer to use to communicate their uncertainty: verbal or numerical. This preference was sometimes personal (“which format do you prefer?”) or more generic (“which format do people prefer?”) (Wallsten, Budescu, Zwick, \& Kemp, 1993). The participants mostly preferred the verbal format of uncertainty communication in this task (Erev \& Cohen, 1990; Wallsten et al., 1993; Xu et al., 2009). With this task, the verbal preference was found across different contexts and in samples from different countries (Brun \& Teigen, 1988; Xu et al., 2009). For instance, 50 out of 66 Norwegian family physicians reported that they would rather use a verbal over a numerical format to inform a patient about the chances of success of a flu vaccine (Brun \& Teigen, 1988) while British family physicians reported a similar preference to inform patients about a drug's side effects (Juanchich \& Sirota, In press).

Format selection tasks provide a clear and consistent finding: people prefer to communicate uncertainty verbally. However, in these tasks, it is hard to know exactly what people prefer and what they focus on to elicit their preference. The generic terminology of the task - verbal vs. numerical - may elicit a focus on a range of verbal and numerical quantifiers and different people may have different quantifiers in mind when answering that question. In fact, when we control for the type of quantifier by specifying one, evidence is more mixed.

In uncertainty phrase selection tasks, participants chose between a verbal and a numerical phrase. In this task, participants sometimes exhibited a verbal preference (Xu et al., 2009) or a numerical one (Erev, Wallsten, \& Neal, 1991; Olson \& Budescu, 1997). Xu et al. (2009) provided participants with a sentence selection task to describe the weather to a family member or friend, in which participants chose between two uncertainty phrases: a phrase featuring a verbal probability and a phrase featuring a single numerical percentage. Most participants selected the verbal uncertainty phrase. In contrast, in a sentence selection task where participants 
could select a verbal phrase from a list or a numerical probability in the $0-100 \%$ range, Olson and Budescu (1997) found the opposite pattern: the participants used a majority of numerical probabilities to convey the likelihood of having the right answer to an almanack question or the probability that a spinner would land on the black area of a black and white pie chart (see also Erev et al. (1991) for a similar verbal preference).

The uncertainty phrase selection tasks outlined above suffer from a key limitation: when participants chose between two uncertainty phrases, the phrases varied in more than the format dimension; they also varied in terms of vagueness. The numerical estimate was precise and the verbal one was vague, thereby confounding the format and the precision of the quantifier. It is therefore unclear if the participants' preference was driven by the level of precision of the quantifiers or by their format. It is indeed possible that people would be more attracted to a numerical format if it was vaguer, such as a range (between 20 and 30\% likely), qualified with a modifier (e.g., around 20\%) or a single bound interval (e.g., more than $20 \%$ likely).

To avoid the confound issue, Du et al. (2013) used a selection task where participants selected a format that was coupled with an example of a phrase that was vague for both the verbal and numerical quantifiers (e.g., verbal: e.g., "possible" or numerical: e.g., "a likelihood of 30\%-40\%"). In these refined selection tasks, on average and across three studies, participants exhibited a numerical preference: the majority selected the numerical interval phrase over the verbal one.

Finally, in general, all the different types of selection tasks (even when vagueness is controlled for) present a challenge: they are not necessarily ecologically valid, meaning that we do not know whether they reflect the way people actually use different uncertainty formats. The materials appeared to be an abbreviated and simplified version of the way people actually use verbal or numerical phrases in real life. For example, uncertainty phrases were often presented 
without grammatical subjects (e.g., Olson \& Budescu, 1997) although we know that the subject of an uncertainty sentence conveys information about the variant of uncertainty that the speaker is experiencing (Juanchich, Gourdon-Kanhukamwe, \& Sirota, 2016; Løhre \& Teigen, 2016). We actually know very little about the properties of uncertainty phrases, so it is difficult to build some selection tasks that are ecological to test the preference for words over numbers: what is being compared is not necessarily what people would actually use.

Taken altogether, the results of the selection tasks did not show a clear picture of the format people prefer to use when conveying uncertainty. People tend to prefer verbal over numerical formats, but contradictory findings and methodological limitations cloud any conclusion. Allowing participants the freedom to decide which format to use, and within this format which level of precision to use, seems the most appropriate way to test the format preference.

Evidence from production tasks. According to evidence from production tasks, people prefer to use verbal phrases. This evidence is, however, limited to only two studies. Erev and Cohen (1990) investigated how four basketball experts predicted 27 basketball events (e.g., UNC will win). Three of the four experts used verbal uncertainty phrases to predict 25 out of 26 basketball outcomes. The fourth expert used some numerical uncertainty phrases for 25 of the 26 predictions. As part of the same study, the authors also asked 21 lay participants to "serve as experts" to predict the outcome of a basketball game; they mostly produced verbal uncertainty phrases $(67 \%)$. A similar preference was replicated with a larger sample size and in two different contexts (next move in chess and the likelihood of having an illness) (Du et al., 2013).

The production tasks provided stronger evidence of a verbal uncertainty preference, but the preference remained mitigated: $20 \%$ to $40 \%$ of the participants actually chose a numerical uncertainty phrase. To account for contrasting findings across tasks and mixed preference, one 
should turn to the reasons why people may or may not prefer verbal uncertainty. The next section reviews the reasons why people tend to prefer one uncertainty format. The review is organised in two families of accounts: accounts which posit a general preference for verbal uncertainty and accounts which posit that the format selection is context-dependent.

\section{Theoretical accounts of the format preference}

The generic preference accounts presuppose that verbal uncertainty has some features that numerical uncertainty lacks. However, the contextual accounts focus on circumstances in which one format would be preferred over the other and hence stress features of verbal and numerical uncertainty that are more appropriate under specific conditions. We argue that the generic accounts cannot explain all the available evidence and that the contextual accounts only focus on separate subsets of findings and that therefore those accounts provide only a scattered representation of the motivation to use verbal and numerical quantifiers. To provide a more unified account of format preference, we built on the commonalities of the three contextual accounts to propose a new account - the uncertainty variant account - which posits that speakers choose an uncertainty format as a function of the variants of uncertainty they experience.

\section{The general preference accounts.}

The naturalness account. According to this account, the way people experience uncertainty can better and more easily be translated into words than numbers (Witteman \& Renooij, 2003; Witteman, Renooij, \& Koele, 2007; Zimmer, 1983). As evidence of this "natural fit", verbal formats of uncertainty are more deeply rooted in the evolutionary history of the human species than numerical uncertainties, which were based on mathematical models elaborated in the 17th century (Zimmer, 1983). However, there are currently no data supporting this assumption. Anecdotal evidence complements this phylogenetic postulate at the ontogenetic level: children 
start by using verbal probabilities and learn numerical probabilities later at school. For example, very few people have heard a three-year-old say that "there is a $50 \%$ probability of rain", but it is not uncommon to hear that "maybe it will rain". The age of acquisition of some probability phrases shows that words are used to quantify uncertainty from a younger age than the numerical uncertainty vocabulary. For example, "maybe" is acquired around 4.5 years old, "likely" at 6.3, "possible" at around 7.3 and "perhaps" around 7.6 years old, whereas the terms "percent" and "percentage" are acquired around 8.2 and 10.3 years old and the term "ratio" at 10.6 years of age (Kuperman, Stadthagen-Gonzalez, \& Brysbaert, 2012). The next generic account makes some more concrete and testable propositions for why the verbal format would be preferred.

The pragmatic account. According to this account, people prefer verbal over numerical uncertainty phrases because they are more informative - they can convey more pragmatic meanings than numerical ones (Hilton, 2008; Moxey \& Sanford, 2000; Teigen \& Brun, 1995; Teigen \& Brun, 2003). Numerical probabilities, for example, do not effectively manifest pragmatic meanings such as the speaker's attitudes towards the outcome occurrence (Teigen \& Brun, 2000). As put by Teigen and Brun: "The numerical probability forecaster may be praised for his apparent expertise, but what exactly is he trying to tell us?” (2003, pp. 142).

In contrast, verbal probabilities convey the implicit attitude of the speaker towards the outcome (Teigen \& Brun, 1995) and "leak" information about past levels of certainty (Juanchich, Teigen, \& Villejoubert, 2010). Other verbal quantifiers, such as verbal frequencies, are also posited to better fulfill similar pragmatic functions than their numerical equivalents (Moxey \& Sanford, 1993, 2000; Moxey, Sanford, \& Dawydiak, 2001). Verbal probabilities also seem more appropriate devices with which to sugarcoat an opinion than numerical probabilities (Kuipers, Moskowitz, \& Kassirer, 1988; Sirota \& Juanchich, 2012; Teigen \& Brun, 2003). For example, "I may have to let you go" would be a more tactful way to say that one made a mistake 
than "there is a $25 \%$ chance that I have to let you go", which would sound unnatural. In the same vein, verbal probabilities are hypothesised to be preferred over numerical probabilities by speakers who use them to avoid being blamed for making an erroneous prediction (Erev \& Cohen, 1990). However, this hypothesis found little support given that the experts in basketball forecasting did not use verbal uncertainty phrases more often when the recipients had a financial stake with which to use their predictions (and would hence be more likely to blame the expert for providing an "incorrect" prediction). The pragmatic account effectively outlines the advantages of using verbal probabilities, but fails to account for situations in which people actually prefer numerical uncertainty (Du et al., 2013; Olson \& Budescu, 1997). For a finer approach to predicting format preference, we turn to the contextual accounts of the format preference.

The contextual accounts. According to these accounts, the format preference is driven by contextual factors. Three contextual hypotheses have been proposed to predict the circumstances in which people prefer verbal uncertainty. The first one is that the preference for verbal uncertainty depends on the role one is playing in a conversation; the second relies on the precision of uncertainty; and the last relies on the animacy of the outcome.

The conversational role hypothesis. Although this paper focuses on the preference of speakers, it is relevant to point out that the role one is taking in a conversation - as speaker or recipient determines people's attitude towards verbal and numerical probabilities. According to this hypothesis, the verbal format is preferred by speakers but not by recipients (Erev \& Cohen, 1990; Wallsten et al., 1993; Xu et al., 2009). Indeed, the same people preferred the verbal format from a speaker's perspective but the numerical one from a recipient's perspective (Brun \& Teigen, 1988; Wallsten et al., 1993). For example, in Wallsten et al. (1993), 45\% of the participants reported that they usually prefer to use a verbal uncertainty when communicating 
their opinions to others, whereas $70 \%$ reported that they would personally prefer to receive numerical information. However, opposite evidence was found in the medical domain. For example, Norwegian family physicians believed that medical risks should be communicated verbally to patients (Brun \& Teigen, 1988). The recipients' preference for numbers was not always replicated either. Among the patients, 35\% preferred to be informed with words, $32 \%$ preferred numbers, $22 \%$ did not have a preference and $8 \%$ preferred a combined verbal/numerical format (Mazur \& Hickam, 1991).

The adaptive decision-maker hypothesis. It has also been postulated that vague assessments are preferred because decision heterogeneity can be beneficial for groups. This stems from the reasoning that if all the members of a group made the same decision, and this decision was incorrect, the group would incur a major loss. However, if only some members of the group made an incorrect decision, the losses experienced by them would be mitigated by the earnings of the other members of the group. Erev et al. (1991) provided some empirical evidence: speakers adapted the format they used to communicate uncertainty as a function of the decision context. In their study, an advisor described a spinner divided in three areas to two observers and three decision-makers, who had to bet on which area the spinner would land. The money earned by the decision-makers was always maximised by a correct bet, whereas the observers and crucially, the advisor - either earned money if all three decision-makers won their bet (control) or if at least one of them won their bet (experimental condition). To maximise their chances of earning money, it was therefore in the advisor's interests for all the decision-makers to choose the same bet in the control group, but to choose different bets in the experimental group. It was expected that advisors would choose to describe the spinner in vaguer terms in the experimental condition - i.e., when it was in their interest that the decision-makers would make different decisions. Overall, participants chose more precise numerical estimates to describe the spinner, 
but, in line with the adaptive decision-maker hypothesis, advisors chose more vague verbal probabilities in the experimental condition than in the control one. The overall preference for numerical estimates in this study could be tied to the fact that the uncertainty was precise, as explained in the next account.

The precision hypothesis. This hypothesis shares the claim of general preference for the verbal format with the naturalness and pragmatic accounts, but it explains the greater frequency of verbal uncertainty by the level of precision of the uncertainty people experience. Participants are expected to prefer the vague verbal format for vague uncertainty and precise numerical formats for precise uncertainty (Hamm, 1991; Olson \& Budescu, 1997; Wallsten, Budescu, \& Erev, 1988; Wallsten et al., 1993). This hypothesis was, for example, developed in a theory of linguistic probabilities which posits that verbal probabilities are always vague and suggests that people choose this format when the uncertainty they experience is vague (Wallsten \& Budescu, 1995). In line with this account, participants selected a numerical probability for a precise uncertainty more often than for a vague one (9 out of 10 times vs. 6 out of 10) (Olson \& Budescu, 1997). In this study the vagueness was manipulated by the quality of the outcome: In the vague uncertainty condition, participants assessed the probability of having the correct answer to an almanac question, whereas in the precise uncertainty condition, they assessed the likelihood that a spinner would land on the white area of a black and white circle. Also, consistent with the precision hypothesis, when given a precise probability through a spinner, participants mostly used a precise numerical probability (between 90\% and 55\%) (Erev et al., 1991).

Taken together with an assumed preference for verbal uncertainty, this hypothesis suggests that people face more situations in which uncertainty is vague, which seems like a reasonable assumption. However, there is no evidence proving that people prefer words over any 
types of numerical estimates, given that this account only used some selection tasks which compared words to precise numerical estimates, therefore confounding format and vagueness. Numbers can also be expressed vaguely, via a range (20-30\%) with a modifier (e.g., "around 20\%") or both (e.g., "around 20-30\%"). Possibly, if they were free to decide the format of communication for their uncertainty, participants would prefer to use vague numerical ranges to convey vague uncertainties.

The animacy hypothesis. This hypothesis posits that the animacy of the uncertain outcome drives the format preference (Du et al., 2013). Animate things (e.g., people, animals) have personal preferences, thoughts, emotions and intentions, in contrast with inanimate things (e.g., lottery balls, coins), which are driven by the law of randomness. Animate uncertainty relies on the dispositions of living beings and was expected to be described more often with verbal uncertainty, than inanimate uncertainty, which relies on randomness (Du et al., 2013). The results were consistent with this expectation: participants chose verbal probabilities to describe the chance of a mouse coming out of 1 of 5 holes in a hollow ball more often than the chances of a ball doing the same (38\% vs. 29\%, Du et al., 2013). The same pattern, albeit stronger, was observed when participants predicted the next move of a person vs. a computer in a game (58\% vs. 17\%). Evidence has shown that the animacy of the uncertainty outcome affects the way people prefer to convey their uncertainty, but it does not really account for people's preference. Indeed, in some of the contexts, more than $50 \%$ of the participants preferred numerical probabilities even for animated outcomes (e.g., the mouse vs. the ball).

\section{The variants-of-uncertainty account.}

Taken on their own, the strong versions of the naturalness and pragmatic accounts cannot explain past empirical findings showing contrasting format preference. If verbal uncertainty was preferred because it is more natural, or for its pragmatic features, this should be the case in any 
context; but this was not so. A weaker version of the general accounts requires the addition of contextual factors: people generally prefer verbal uncertainty, except in some specific circumstances. Existing contextual accounts provide a complementary, yet scattered, picture of the reasons why people prefer verbal uncertainty. We propose here a new account that unifies the contextual accounts and which describes with parsimony the circumstances in which people prefer verbal uncertainty.

The common point of all the contextual accounts of uncertainty format preference is that they all mention, or allude to, variants of uncertainty as a determinant (Du et al., 2013; Olson \& Budescu, 1997; Wallsten et al., 1993). Building on previous findings and theoretical accounts of uncertainty format preference, we propose that the variants of uncertainty are a key determinant of people's format preference in uncertainty communication.

Uncertainty theorists generally agree that variants of uncertainty exist, although their numbers and nature vary across different classifications (Hacking, 1966; Howell \& Burnett, 1978; Kahneman \& Tversky, 1982; Keren \& Teigen, 2001; Lagnado \& Sloman, 2007; Ülkümen, Fox, \& Malle, 2016). Those variants reflect different psychological realities and obey different rules leading to different decision preferences (e.g., Brun \& Teigen, 1990; Robinson, Pendle, Rowley, \& Beck, 2009). For example, participants were found to prefer betting with a mixture of internal and external uncertainty rather than just internal uncertainty - supposedly because they would not bear all of the responsibility for not being correct (Brun \& Teigen, 1990).

We focus here on three variants of uncertainty inspired by Kahneman and Tversky (1982) and Keren and Teigen (2001): (i) epistemic uncertainty that stems from lack of knowledge, (ii) distributional uncertainty that stems from the world and relies on the frequency of similar outcomes in the past and (iii) dispositional uncertainty, that relies on a causal assessment and which can either stem from personal predispositions or from the world. The 
variant of uncertainty account posits that people prefer to use verbal uncertainty for epistemic and dispositional uncertainty stemming from personal predispositions and numerical uncertainty for distributional outcomes and dispositional uncertainty stemming from the world.

The findings of the contextual accounts of the format preference can be unified within a variant of uncertainty account. Olson and Budescu (1997) manipulated the precision of the probability that people could elicit, but discuss the fact that this manipulation had implications for the variants of uncertainty that people experienced. In their manipulation, the vague uncertainty was also internal (it relied on a lack of knowledge regarding the almanac question) whereas the spinner probability was distributional (it relied on the portion of white in the black circle). Further, people report that they prefer numerical estimates when they can compute the probability (Wallsten et al., 1993), as can be the case with distributional uncertainty. Finally, the animacy findings can be reinterpreted in the uncertainty variant framework. The difference was framed as animacy vs. inanimacy, but we could also consider that the ball triggered a distributional uncertainty, whereas the mouse evoked a more dispositional uncertainty: the chances that the mouse would choose a specific hole relied on the disposition of a living being (be it a mouse). Ülkümen et al. (2016) provided indirect evidence of the role of the source regarding format preference. In a corpus study, the authors showed that verbal quantifiers characterising an aleatory uncertainty (e.g., likelihood, probability) were more often qualified with a numerical probability than verbal quantifiers characterising an epistemic uncertainty (e.g., sure, certainty).

\section{The present research}

The present research focused on two aims. First, to accumulate ecologically valid evidence on the way speakers naturally prefer to communicate their uncertainty. Our approach kept the methodological strengths of previous studies by employing a production paradigm (Du 
et al., 2013; Erev \& Cohen, 1990), a fairly large sample size (Du et al., 2013) and a range of outcomes that reflected different uncertainties (Olson \& Budescu, 1997). Participants either communicated their uncertainty orally (Experiment 1) or through writing (Experiments 2 and 3). This methodology has the potential to bring forth new and more definitive evidence regarding the format that people prefer to use.

The second aim of the present research was to test the hypotheses derived from the variants-of-uncertainty account. We hypothesised that participants would prefer verbal uncertainty phrases over numerical ones for epistemic uncertainties and for internal dispositional uncertainty. In contrast, we hypothesised that participants would prefer numerical probabilities for distributional uncertainties triggered by outcomes that have a clear set of alternative outcomes and for uncertainty stemming from causal dispositions of the world.

Method statement. The materials and data from the three experiments are available on the Open Science Framework:

https://osf.io/x8uh9/?view_only=d7989e0553ee4cd1b0da4e15d1e5a8fc.

\section{Experiment 1}

Participants communicated their uncertainty regarding six outcomes selected to reflect three variants of uncertainty. The participants communicated their uncertainty orally, in an interviewstyle study in Experiment 1.

Participants. Overall, 55 participants took part in a short interview conducted by one of two research assistants. We excluded data from seven participants who did not follow the instructions to complete the study. Age ranged from 19 to 50 years $(M=28.18, S D=8.94), 45 \%$ female. All participants reported being fluent in English with education levels ranging from $\mathrm{O}$ levels to postgraduate studies. 
Design. A within-subjects design was employed by crossing 3 variants of uncertainty (epistemic, dispositional and distributional) *2 (outcomes); thus, the participants communicated their uncertainty regarding 6 outcomes.

Procedure and materials. Participants imagined that the interviewer was answering a quiz and they had to help him/her assess whether or not a fact was true or the chances that an event would occur (two RAs shared the task: a female and a male). Participants communicated their uncertainty regarding the pairs of outcomes presented in one of three set orders (epistemic, dispositional, distributional; distributional, epistemic, dispositional; or dispositional, distributional, epistemic).

The variants of uncertainty were manipulated through the nature of the outcome. The list of outcomes is presented in Table 1. For epistemic uncertainty, we selected two outcomes which required knowledge to achieve certainty (e.g., "Is the Luster a Norwegian fish?”) For dispositional uncertainty, we selected two outcomes for which the degree of certainty relied on the disposition of a person (e.g., "Would someone give his blood when asked by a neighbour?") Finally, for distributional uncertainty, we selected two outcomes that had a clear set of alternative events based on some physical characteristics of the world (e.g., "Obtaining an even score with a six-sided die").

A separate experiment (hereafter, Experiment A) was conducted to assess the extent to which people perceived the different outcomes to reflect the different variants of uncertainty. The study was conducted online via Amazon Mechanical Turk with 70 American participants (age: $M=34.01, S D=9.34$ years; $57 \%$ males, $60 \%$ with at least a two-year college degree). Participants were informed that uncertainty can be caused by different factors that correspond to the three variants of uncertainty we focused on: "Uncertainty can be caused by a lack of knowledge (e.g., "I am not sure whether the Eiffel Tower is more than 100 metres high"); 
"Uncertainty can be caused by the stable physical properties of the world (e.g., "A coin has two sides and therefore has a 50\% chance of falling on tails"); and "Uncertainty can be caused by a person's disposition (e.g., "I may or may not have a coffee this morning according to my mood"). Participants read the list of six outcomes (presented in a randomised order to each participant) and had to match the outcome with the most likely cause of uncertainty in the three listed above (summarised as "Lack of knowledge", "Stable physical properties of the world" and "Disposition of a person"). The results indicated that the outcomes were perceived as being caused by different factors, hence reflecting variants of uncertainty. As shown in Table 1, most of the participants perceived that uncertainty regarding the two epistemic outcomes was caused by a lack of knowledge (90\%), uncertainty regarding the two dispositional outcomes was believed to be caused by the disposition of a person (78\%) and uncertainty regarding the two distributional outcomes was believed to be caused by the physical characteristics of the world (89\%). The perception of the cause within a pair of outcomes with the same expected variant of uncertainty was judged as stemming consistently from the same cause. 
Table 1.

Perceived cause of the pairs of outcomes expected used in Experiments 1 and 2 to reflect an epistemic, dispositional and distributional uncertainty (Experiment $A, N=70$ ).

Perceived cause

\begin{tabular}{|c|c|c|c|c|}
\hline \multirow{3}{*}{$\begin{array}{l}\text { Expected } \\
\text { variant }\end{array}$} & \multirow{3}{*}{ Outcome } & \multirow{3}{*}{$\begin{array}{l}\text { Lack of } \\
\text { knowledge }\end{array}$} & \multirow{3}{*}{$\begin{array}{l}\text { Disposition of } \\
\text { a person }\end{array}$} & \multirow{3}{*}{$\begin{array}{l}\text { Stable physical } \\
\text { properties of the } \\
\text { world }\end{array}$} \\
\hline & & & & \\
\hline & & & & \\
\hline \multirow[t]{2}{*}{ Epistemic } & $\begin{array}{l}\text { Uncertainty that the Luster is } \\
\text { a Norwegian fish. }\end{array}$ & $89 \%$ & $3 \%$ & $9 \%$ \\
\hline & $\begin{array}{l}\text { Uncertainty that Reynes is a } \\
\text { small village in France. }\end{array}$ & $91 \%$ & $1 \%$ & $7 \%$ \\
\hline \multirow[t]{2}{*}{ Dispositional } & $\begin{array}{l}\text { Uncertainty that someone } \\
\text { would become a blood donor } \\
\text { when asked by a neighbour. }\end{array}$ & $11 \%$ & $77 \%$ & $11 \%$ \\
\hline & $\begin{array}{l}\text { Uncertainty that someone } \\
\text { would bungee jump from a } \\
\text { bridge if asked by a friend. }\end{array}$ & $7 \%$ & $79 \%$ & $14 \%$ \\
\hline \multirow[t]{2}{*}{ Distributional } & $\begin{array}{l}\text { Uncertainty that someone } \\
\text { obtains an even score with a } \\
\text { six-sided die. }\end{array}$ & $4 \%$ & $4 \%$ & $91 \%$ \\
\hline & $\begin{array}{l}\text { Uncertainty that someone } \\
\text { selects a heart from a deck of } \\
\text { cards. }\end{array}$ & $7 \%$ & $6 \%$ & $87 \%$ \\
\hline
\end{tabular}

The interviews were transcribed verbatim and the full answer to each question was scrutinised. The format was coded as numerical if the response featured at least one numerical 
expression of frequency, probability or the number of alternative outcomes. The detailed coding scheme with some example sentences is available in Appendix I.

Two research assistants, blind to the hypotheses of the study, coded the format for each of the six sentences (0: numerical and 1: verbal). The inter-rater reliability was good: Cohen's kappas were between .0 .95 and 1 with a median of 1 . Most participants chose the same format to convey uncertainty within a same variant (e.g., numerical for the two distributional outcome, verbal for the two dispositional outcomes), so we decided to use a sum score of verbal preference per variant of uncertainty. In the three experiments presented here, we used the sum number of the verbal format per uncertainty variant as the verbal format preference variable. The sum variable is ordinal and ranged from 0 to 2 , where a score of 0 indicated that the participant used numbers to describe their uncertainty for both outcomes within that variant, and a score of 2 meant that the participant used only words to describe their uncertainty for both outcomes within that variant. We describe the format preference using percentage of verbal format per outcome within a variant. We used the verbal sum score to derive a percentage of verbal preference for each variant of uncertainty and for each participant: A score of 2 (out of 2) was translated into a verbal preference percentage of $100 \%$ and a score of 0 was translated into a percentage of $0 \%$ while a score of 1 was translated into a score of $50 \%$. We tested the effect of the variant of uncertainty on the format preference variable using a non-parametric Friedman ANOVA, while the post-hoc comparisons were conducted with three Wilcoxon tests.

\section{Results}

\section{Effect of variants of uncertainty on format preference}

Participants produced a majority of verbal uncertainty phrases to describe the epistemic and dispositional uncertainties but used more numerical phrases for the distributional uncertainty: $83 \%$ and $75 \%$ vs. $36 \%$. The uncertainty variants had a statistically significant effect on format 
preference, Friedman ANOVA $\chi^{2}(2, N=55)=47.06, p<.001$. We complemented this analysis with three Wilcoxon signed rank tests. The results showed that epistemic and dispositional uncertainty were more often conveyed using words than distributional uncertainty and that epistemic and dispositional uncertainties did not differ statistically, respectively, $Z=-5.17, p<$ $.001, Z=-4.45, p<.001$ and $Z=-1.79, p=.074$.

Table 2.

Proportion of verbal format used as a function of the variant of uncertainty in Experiment 1, 2 and 3. Participants chose more verbal format for the epistemic and dispositional outcomes than for the distributional ones.

\begin{tabular}{lccc}
\hline \multicolumn{4}{c}{$\%$ Verbal format } \\
\hline Epistemic & Experiment 1 $(N=55)$ & Experiment 2 $(N=201)$ & Experiment 3 $(N=220)$ \\
French village & $80 \%$ & $69 \%$ & $43 \%$ \\
Norwegian fish & $86 \%$ & $69 \%$ & $44 \%$ \\
Total & $83 \%$ & $70 \%$ & $42 \%$ \\
Dispositional (animate) & & & \\
Blood donation & $76 \%$ & $67 \%$ & $47 \%$ \\
Bungee jumping & $73 \%$ & $66 \%$ & $41 \%$ \\
Total & $75 \%$ & $66 \%$ & $41 \%$ \\
Dispositional (inanimate) & & & \\
Tyre & -- & -- & $39 \%$ \\
Migraine & -- & -- & $37 \%$ \\
total & -- & -- & $42 \%$ \\
Distributional & & & \\
Die & $38 \%$ & $24 \%$ & $36 \%$ \\
Cards & $35 \%$ & $36 \%$ & $34 \%$ \\
Total & $36 \%$ & $30 \%$ & $41 \%$ \\
& & & $42 \%$ \\
\hline Overall total & $65 \%$ & $55 \%$ & \\
\hline
\end{tabular}

Note: In Experiment 1, participants produced their sentences orally, but wrote them for Experiment 2 and 3. In Experiment 3, the uncertainty was precise for all the outcomes through a pie chart. In Experiment 3 the average format per source varies from the individual outcome because we did not include the missing data in the average number of verbal format per 
uncertainty variant (e.g., if one sentence was excluded out of the two, we did not include this in the total percent variable).

In line with the variants-of-uncertainty account, the variants of uncertainty had an effect on the format of uncertainty produced by the participants. The participants used words for the epistemic and dispositional outcomes and numbers for the distributional outcomes.

\section{Experiment 2}

In Experiment 2 we replicated Experiment 1, but instead of being interviewed and providing their answers orally, participants completed an online survey in which they wrote down their answers.

\section{Method}

Participants. A total of 201 Amazon Mechanical Turk workers took part in a five-minute survey in exchange for a $\$ 0.40$ payment. After inspection of the data we noticed that six participants consistently did not follow the instructions - e.g., answering the questions with random or irrelevant text (e.g., "I like to fly.. i love being above the clouds"; "Nothing too odd about wearing shorts in the winter"). Their data was excluded from the analyses. Out of the remaining 194 participants (age: $M=37.88, S D=24.21$ years), $43 \%$ were women, $77 \%$ had at least some higher education and were mostly White Caucasian (77\%).

Design, Materials and Procedure. This study formally replicated Experiment 1 in a written format. Data was coded as that of Experiment 1 . The inter-coder reliability was satisfactory (Cohen's kappas were between .85 and $1 ; M d n=1$ ). 


\section{Results}

\section{Effect of uncertainty variants on format preference}

As shown in Table 2, the participants produced a majority of verbal uncertainty phrases to describe the epistemic or dispositional uncertainties and produced more numerical uncertainty phrases for the distributional uncertainty. A Friedman ANOVA showed a significant effect of uncertainty variants on format, $\chi^{2}(2, N=193)=135.92, p<.001$. Wilcoxon pairwise comparisons showed that the epistemic and dispositional uncertainties were more often conveyed using words than the distributional uncertainty, and that the epistemic and dispositional uncertainties did not differ, $Z=-8.34, p<.001, Z=-7.84, p<.001$ and $Z=-1.54$, $p=.124$. The results replicated the effect of the uncertainty variants on format preference found in Experiment 1. The participants used words for the epistemic and dispositional outcomes and numbers for the distributional outcomes.

The results of Experiments 1 and 2 show that the epistemic and dispositional uncertainties were communicated in a similar fashion, whereas the distributional uncertainty was different. This pattern of finding is also consistent with the view that the format depends on the precision of uncertainty, given that it is easy to derive a precise probability for the two distributional outcomes, whereas it is not possible for the dispositional and epistemic outcomes.

\section{Experiment 3}

In this experiment, we replicated Experiment 2 while introducing three important changes that aimed to further disentangle the precision account and the variants-of-uncertainty account. First, we included two new dispositional outcomes that related to the disposition of the world rather than to the disposition of a person. So far, the dispositional uncertainty tested in Experiments 1 and 2 was internal: whether a person becomes a blood donor or decides to bungee 
jump. However, dispositional uncertainty can also be external: when the causal reasons belong to the world. To further put the hypothesis of the variants-of-uncertainty to the test, we compared outcomes that have internal dispositional uncertainty (as in Experiments 1 and 2) and outcomes that have external dispositional uncertainty. We expected epistemic markers to be more common with internal disposition and epistemic uncertainty than for external disposition uncertainty and distributional uncertainty.

Second, we provided the participants with a precise estimate of the degree of certainty that they should communicate for all the outcomes. So far, the epistemic and dispositional outcomes were related to a vague uncertainty and the distributional outcome to a precise one: hence, precision was a natural confounding variable. One alternative explanation for our previous findings is therefore that the precision of uncertainty - which naturally co-occurs with the variant of uncertainty - had an impact on format preference. In the present experiment, we tested whether the variants of uncertainty had an effect on format preference over and above precision by keeping precision constant across all of the outcomes.

Third, the outcomes were presented in a random order, whereas before they were randomly presented within an outcome category: epistemic outcomes, distributional and dispositional outcomes were presented together, possibly re-enforcing the similarities of expression of the variant of uncertainty triggered by the pair of outcomes.

\section{Method}

Participants. A total of 220 Amazon Mechanical Turk workers took part in a five-minute survey in exchange for a $\$ 0.40$ payment. Age ranged from 19 to $66(M=37.24, S D=11.01)$, 42\% were women, $77 \%$ had at least some higher education and $78 \%$ were White Caucasian. After inspection of the data we decided to exclude the sentences that did not make sense instead of whole cases (9\% for the Reynes and Die outcome to $13 \%$ for the headache outcome). 


\section{Design, Materials and Procedure}

We used the same materials as in Experiments 1 and 2 with two changes: (i) we tested two additional outcomes that represented an inanimate dispositional uncertainty. The two new uncertain outcomes were the tyre and migraine outcomes: "A tyre puncture after driving on broken glass" and "A new headache treatment works against bacterial infections". The second change was that for each outcome we provided a pie chart depicting a precise level of certainty.

An experiment was conducted separately - Experiment B: $N=50$ Amazon Mechanical Turk workers (age: $M=34.86, S D=8.12$ years; $67 \%$ males, $80 \%$ with at least a two-year college degree). This experiment assessed the cause of the uncertainty associated with the eight outcomes and its relative contribution to each pair of outcomes. The study was the same as Experiment A except for the two additional external dispositional outcomes and the new source of uncertainty: participants were told that uncertainty could also be caused by the disposition of the world (e.g., "A car may or may not break down according to how well it has been maintained").

As shown in Table 3, most of the participants perceived that uncertainty regarding the two epistemic outcomes was caused by a lack of knowledge (76\%), uncertainty regarding the two internal dispositional outcomes was believed to be caused by the disposition of a person $(84 \%)$ and uncertainty regarding the two distributional outcomes was believed to be caused by the physical characteristics of the world (79\%). However, the perception of the external dispositional uncertainty was not so clear cut: a small majority selected the cause "disposition of the world" for the tyre outcome but only $28 \%$ chose it for the chances of a new headache treatment working against bacterial infections. Nevertheless, the headache treatment outcome can be considered to be referring more to the disposition of the world given that participants selected this answer more often than for the outcomes referring to other variants of uncertainty: 
four times more than for the epistemic outcomes, seven times more than for the animate dispositional outcomes and three times more than for the distributional outcomes.

Table 3. Perceived cause of the pairs of outcomes expected used in Experiment 3 to reflect an epistemic, dispositional animate, dispositional inanimate and distributional uncertainty (Experiment $B, N=50$ ).

\begin{tabular}{llllll}
\hline \multicolumn{1}{l}{} & \multicolumn{5}{l}{ Perceived cause } \\
\hline $\begin{array}{llll}\text { Expected } \\
\text { variant }\end{array}$ & Outcome & $\begin{array}{l}\text { Lack of } \\
\text { knowledge }\end{array}$ & $\begin{array}{l}\text { Disposition of } \\
\text { a person }\end{array}$ & $\begin{array}{l}\text { Disposition of } \\
\text { the world }\end{array}$ & $\begin{array}{l}\text { Stable physical } \\
\text { properties of the } \\
\text { world }\end{array}$ \\
\hline Epistemic & Luster & $\mathbf{7 1 \%}$ & $5 \%$ & $6 \%$ & $4 \%$ \\
& Reynes & $\mathbf{8 0 \%}$ & $10 \%$ & $8 \%$ & $2 \%$ \\
$\begin{array}{l}\text { Dispositional } \\
\text { animate }\end{array}$ & Blood & $2 \%$ & $\mathbf{8 8 \%}$ & $4 \%$ & $6 \%$ \\
& $\begin{array}{l}\text { Bungee } \\
\text { jump }\end{array}$ & $4 \%$ & $\mathbf{8 0 \%}$ & $4 \%$ & $12 \%$ \\
$\begin{array}{l}\text { Dispositional } \\
\text { inanimate }\end{array}$ & Tyre & $8 \%$ & $33 \%$ & $\mathbf{5 5 \%}$ & $33 \%$ \\
& Headache & $51 \%$ & $12 \%$ & $\mathbf{2 8 \%}$ & $10 \%$ \\
Distributional & Die & $12 \%$ & $4 \%$ & $8 \%$ & $\mathbf{7 7 \%}$ \\
& Card & $4 \%$ & $8 \%$ & $8 \%$ & $\mathbf{8 0 \%}$ \\
\hline
\end{tabular}

The probability of each outcome was either $25 \%$ or $50 \%$ and was depicted in a pie chart (with the probability of the outcome being correct in black and the remaining section in white). We chose these two probability magnitudes because they roughly matched the reported level of certainty of participants in Experiments 1 and 2. The outcomes were presented on different pages and in a random order for each participant. The introductory example was changed and focused on the chances of President Trump being re-elected (with a 10\% probability). A checking question placed at the end of the study asked participants the numerical probability depicted in the graph to check that it was perceived to be precise. This was indeed the case, with 
$99 \%$ of the participants correctly identifying the $50 \%$ proportion and $93 \%$ selecting the $25 \%$ proportion in the pie chart. The participants' answers were coded by a single research assistant using the same coding framework as in Experiments 1 and 2. In this experiment a range of responses were clearly not following the instructions (e.g., "You are democrat than", "Look man, the last 3 rolls were odd").

\section{Results}

\section{Effect of uncertainty variants on format preference}

Having a precise estimate swayed participants' preference from verbal to numerical with $42 \%$ of participants overall using a verbal form of uncertainty across the outcomes (Table 3). A Friedman ANOVA showed that the effect of the uncertainty variants on format was above the statistical significance threshold, $\chi^{2}(3, N=183)=6.25, p=.104$. However, as in Experiments 1 and 2, pairwise comparisons showed that epistemic and internal dispositional uncertainty were more often conveyed using words than distributional uncertainty, $Z=-2.25, p=.024$ and $Z=-$ $1.98, p=.048$. Epistemic uncertainty did not differ from animate and inanimate dispositional uncertainty and the two dispositional uncertainty did not differ, $Z=-0.39, p=.706$ and $Z=-$ $1.44, p=.151, Z=-0.92, p=.357$. Finally, inanimate dispositional uncertainty and distributional uncertainty did not differ $Z=-0.88, p=.379$.

\section{Discussion}

Which format do people prefer when conveying their uncertainty? It is commonly cited that people prefer to express their uncertainty in words, but past empirical evidence does not provide unequivocal support for this assertion. We aimed to provide some direct and ecological valid evidence regarding this preference. Several accounts have provided hypotheses regarding why people prefer verbal uncertainty (e.g., a more intuitive format, richer pragmatic meaning) and the 
circumstances in which this preference may be altered (e.g., conversational role, outcome animacy, uncertainty precision). We have built on existing empirical findings and theoretical accounts to propose that people choose format as a function of the variant of uncertainty that they experience.

\section{New and robust evidence of verbal probability preference.}

Across these experiments, almost 500 participants have communicated their uncertainty regarding six or eight outcomes, producing a total of more than 3,000 sentences. Overall, and based on this sample of outcomes, the data indicate that people tend to prefer verbal expressions of uncertainty. By using a production task and a range of outcomes, our studies provide some more ecologically valid evidence regarding the format that people prefer to convey their uncertainty. In the selection tasks, the task was either too vague (do you prefer a verbal format?), allowing too much room for participants to use their imagination (Brun \& Teigen, 1988; Erev \& Cohen, 1990; Wallsten et al., 1993; Xu et al., 2009) or too specific (do you prefer "it is possible" or "it is $40 \%$ probable"?), thereby constraining the participants' choice and possibly confounding format and precision. Allowing participants the freedom to decide which format to use and, within this format, which level of precision to use, complemented methodologies commonly used to test the format preference (Erev et al., 1991; Olson \& Budescu, 1997; Xu et al., 2009). Our results are in line with trends observed in format selection tasks (Brun \& Teigen, 1988; Erev \& Cohen, 1990; Wallsten et al., 1993; Xu et al., 2009), providing some evidence of their validity. The results also complement past work using a production task (Du et al., 2013; Erev \& Cohen, 1990) by focusing on new outcomes.

One clear limitation of our approach is that focusing on whole phrases produced by participants requires a coding procedure that relies on definitional assumption. For example, a numerical uncertainty included any sentence that featured a number, whether it was to describe a 
frequency (e.g., $50 \%$ of the numbers are odd on a die); a number of outcomes (there are 3 odd numbers on a die); and, in addition, the coding procedure is subject to interpretation, as shown by some inter-coder variability.

Further, thanks to a focus on different response modality, our studies hint that modality affects format preference. Results from Experiment 1 - based on an oral expression of uncertainty - featured more verbal uncertainty than the results in Experiment 2 - based on a written expression of uncertainty. We did not have a set hypothesis regarding a possible effect of modality but, in hindsight, we could have expected that people would use more verbal formats in conversation than in writing (which was the case), given the expected intuitive nature of verbal uncertainty and that people would be more spontaneous orally than in writing.

\section{Accounts of the format preference - variants of uncertainty vs. precision?}

Our results show that people choose a format as a function of the variants of uncertainty. In three experiments, employing a methodology maximising ecological validity, we found evidence that participants select a format as a function of the variant of uncertainty. The participants exhibited a clear preference for verbal over numerical formats for epistemic and dispositional uncertainty and for numerical uncertainty over verbal for distributional uncertainty when we did not control for the precision of uncertainty. When precision was kept constant however, the participants were still sensitive to the variants of uncertainty, but they mostly used a numerical quantifier of uncertainty. The variation in verbal format was possibly reduced because the precise estimates were provided by a computer, which is believed to be an external source of uncertainty.

The epistemic and dispositional uncertainties were equally often conveyed in words. Therefore, a more parsimonious account of uncertainty format preference could only contrast distributional uncertainty with the other variants. Our results regarding the role of the variants of 
uncertainty do not correspond well with a tri-partite model of the variants, but with a more recent, dual-model of variants of uncertainty (Ülkümen et al., 2016), which features epistemic and aleatory uncertainty. In this account, epistemic uncertainty includes both epistemic uncertainty, as defined by Kahneman and Tversky (1982), and dispositional uncertainty, as defined by Keren and Teigen (2001), while the aleatory uncertainty maps onto our distributional uncertainty. Further research should test whether this framework could best explain the preference for words and numbers in uncertainty communication.

Our results also indirectly lend support to the precision hypothesis, which posits that people prefer verbal formats for vague uncertainty and numerical formats for precise uncertainties (Hamm, 1991; Olson \& Budescu, 1997; Wallsten et al., 1993). Indeed, in Experiments 1 and 2, the source of uncertainty is clearly connected with its vagueness: epistemic uncertainty being vague and distributional uncertainty precise. The effects of the source observed when source and precision were disentangled in Experiment 3 were smaller, indicating that the larger effects observed in Experiments 1 and 2 were likely partly driven by the precision of uncertainty. However, evidence from Experiment 3 showed that variants mattered even after we controlled for the level of precision of uncertainty. In addition, when uncertainty was precise, participants did not show a strong preference for the numerical format - around 55\% did. Altogether, it seems that both the variants of uncertainty and the precision of the probability contribute to the format preference.

\section{Is verbal uncertainty more natural?}

Findings suggest that, in general, people prefer to convey uncertainty regarding their knowledge or facts that have a causal underpinning in words, whereas they prefer to communicate uncertainty regarding distributional events in numbers. Given that there are a limited number of events for which a precise probability can be computed (besides a die and a card draw), most 
events rely to some extent on knowledge or causal factors (e.g., in a race, not all horses can be considered as having the same chance of winning; factors such as the skills of the jockey or the health of the horse on that day will influence the perception of the chance of a particular horse winning the race).

This supports the view that verbal uncertainty more effectively represents the way people feel uncertainty than numerical uncertainty phrases, as posited in the naturalness account (Zimmer, 1983). It is also in line with the finding that speakers prefer verbal uncertainty phrases (Erev \& Cohen, 1990; Xu et al., 2009) and that they are common in natural language corpus (Neuner-Jehle et al., 2011), and in some production studies (Du et al., 2013; Erev \& Cohen, 1990). Given that they are used more often, we can also assume that verbal uncertainty phrases may be perceived to be more familiar and hence easier to process as suggested by Witteman and Renooij (2003).

The preference for a given format does not only have theoretical value. The format people use and the reason why they use it may influence recipients' judgments and subsequent decisions. For example, participants expressed more trust and felt more comfortable with a GP providing a numerical estimate for their risk of having prostate cancer (Gurmankin, Baron, \& Armstrong, 2004) and greater intention to adhere to a treatment when side effects were presented numerically instead of verbally (Young \& Oppenheimer, 2009). From a practical perspective, individuals routinely providing forecasts, prognoses or predictions should be aware that, based on the uncertainty variant associated with the outcome they describe, they will use a particular form of language and that this language is consequential. It has many attributes that affects recipients' perceptions and decisions, such as the format, but also the type of grammatical subject (Juanchich et al., 2016) and the presence of reasons (Flugstad \& Windschitl, 2003). 


\section{Conclusion}

Our results point towards the adaptability of people in different contexts: they prefer to use verbal uncertainty for epistemic and dispositional outcomes but numerical certainty for distributional outcomes. Assuming that there are more outcomes for which one cannot derive a precise probability (e.g., a known number of alternative outcomes and their frequencies), evidence supports the fact that people use more verbal uncertainty phrases than numerical ones and that they may therefore be more natural and easier to process. The variants of uncertainty mattered over and above the precision of uncertainty although it reduced their effect. 


\section{Compliance with ethical standards}

\section{Conflict of interest}

Marie Juanchich declares that she has no conflict of interest. Miroslav Sirota declares that he has no conflict of interest.

\section{Ethical approval}

All procedures performed in the experiments reported here involving human participants were conferred a favourable opinion from University of Essex's Department of Psychology Research Ethics Committee and were in accordance with the British Psychological Society Code of Ethics and Conduct and with the 1964 Helsinki Declaration and its later amendments.

\section{Informed consent}

Informed consent was obtained from all individual participants included in the three experiments reported here. 


\section{Appendices}

\section{Appendix I}

Coding instructions for Experiments 1 and 2

Format Whether the response features some numbers or not.

1: Verbal only.

0 : Feature at least one numerical expression of frequency, probability or the number of alternative outcomes), even written in words. Mathematical operands were not counted as numerical (e.g., "Half the time" $\rightarrow$ verbal).

- Example of sentence coded 1: "It is quite probable that Reynes is a small French village."

- Example of sentence coded 0: "There is a fifty-fifty chance that Reynes is a small village in France." 


\section{References}

Almond, L., Alison, L., \& Porter, L. (2007). An Evaluation and Comparison of Claims Made in Behavioural Investigative Advice Reports Compiled by the National Policing Improvements Agency in the United Kingdom. Journal of Investigative Psychology and Offender Proffiling, 4, 71-83.

Brun, W., \& Teigen, K. H. (1988). Verbal probabilities: Ambiguous, context dependent, or both? Organizational Behavior and Human Decision Processes, 41, 390-404. doi: http://dx.doi.org/10.1016/0749-5978(88)90036-2

Brun, W., \& Teigen, K. H. (1990). Prediction and postdiction preferences in guessing. Journal of behavioral Decision Making, 3, 17-28. doi: http://dx.doi.org/10.1002/bdm.3960030103

Budescu, D. V., \& Wallsten, T. S. (1995). Processing linguistic probabilities: General principles and empirical evidence. In R. H. J.R. Busemeyer \& D. Medin (Eds.), (pp. 275-318): Amsterdam: Academic Press.

Clark, D. A. (1990). Verbal uncertainty expression : A critical review of two decades of research. Current psychology: Research and reviews, 9, 203-235. doi: http://dx.doi.org/10.1007/BF02686861

Collins, S., \& Alison, L. (2002). How certain are offender profilers about the claims they make?

Dhami, M. K., Mandel, D. R., Mellers, B. A., \& Tetlock, P. E. (2015). Improving intelligence analysis with decision science. Perspectives on Psychological Science, 10, 753-757.

Du, X.-L., Liu, S.-H., Xu, J.-H., Rao, L.-L., Jiang, C.-M., \& Li, S. (2013). When uncertainty meets life: The effect of animacy on probability expression. Judgment and Decision Making, 8, 425-438.

Erev, I., \& Cohen, B. L. (1990). Verbal versus numerical probabilities: Efficiency, biases, and the preference paradox. Organizational Behavior and Human Decision Processes, 45, 118. doi: http://dx.doi.org/10.1016/0749-5978(90)90002-Q 
Erev, I., Wallsten, T. S., \& Neal, M. M. (1991). Vagueness, ambiguity, and the cost of mutual understanding. Psychological Science, 2, 321-324. doi: 10.1111/j.14679280.1991.tb00159.x

European Commission. (1998). A guideline on the readability of the label and package leaflet of medicinal products for human use.

European Food Safety Authority. (2017). Guidance on Uncertainty in EFSA Scientific Assessment draft.

Flugstad, A. R., \& Windschitl, P. D. (2003). The influence of reasons on interpretations of probability forecasts. Journal of behavioral decision making, 16, 107-126. doi: 10.1002/bdm.437

Gurmankin, A. D., Baron, J., \& Armstrong, K. (2004). The effect of numerical statements of risk on trust and comfort with hypothetical physician risk communication. Medical Decision Making, 24, 265-271. doi: 10.1177/0272989X04265482

Hacking, I. (1966). Subjective Probability. British Journal for the Philosophy of Science, 16.

Hamm, R. M. (1991). Selection of Verbal Probabilities: A Solution for Some Problems of Verbal Probability Expression. Organizational behavior and human decision processes, $48,193-223$.

Hilton, D. J. (2008). Emotional tone and argumentation in risk communication. Judgment and Decision Making, 3, 100-110.

Ho, E. H., Budescu, D. V., Dhami, M. K., \& Mandel, D. R. (2015). Improving the communication of uncertainty in climate science and intelligence analysis. . Behavioral Science \& Policy, 1 .

Homeland Security Risk Steering Committee. (2008).

Department of Homeland Security Risk Lexicon: Homeland Security. 
Honda, I., \& Yamagishi, K. (2009). Perceived certainty based on verbal probability phrases: Effect of directionality and its dependence on method. Japanese Psychological Research, $51,266-273$.

Howell, W. C., \& Burnett, S. A. (1978). Uncertainty measurement: A cognitive taxonomy. Organizational Behavior and Human Performance, 22, 45-68.

Intergovernmental Panel on Climate Change. (2007). Climate change 2007. The climate change physical science Basis.

Intergovernmental Panel on Climate Change. (2013). Fifth Assessment Report Climate Change 2013: The Physical Science Basis. Summary for Policymakers.

International Accounting Standard Committee. (1998). Provisions, Contingent Liabilities and Contingent Assets, International Accounting Standard 37. London.

Juanchich, M., Gourdon-Kanhukamwe, A., \& Sirota, M. (2016). 'I am uncertain’ or ‘It is uncertain'? How linguistic markers of the uncertainty source affect uncertainty communication. Journal of Memory and Language.

Juanchich, M., \& Sirota, M. (In press). Most family physicians report communicating the risks of adverse drug reactions in words, but less so for severe ones. Applied Cognitive Psychology.

Juanchich, M., Sirota, M., \& Butler, C. L. (2012). The perceived functions of linguistic risk quantifiers and their effect on risk, negativity perception and decision making. Organizational Behavior and Human Decision Processes, 118, 72-81. doi: http://dx.doi.org/10.1016/j.obhdp.2012.01.002

Juanchich, M., Teigen, K. H., \& Villejoubert, G. (2010). Is guilt 'likely' or 'not certain'? Contrast with previous probabilities determines choice of verbal terms. Acta Psychologica, 135, 267-277. doi: 10.1016/j.actpsy.2010.04.016 
Kahneman, D., \& Tversky, A. (1982). Variants of uncertainty. Cognition, 11, 143-157. doi: http://dx.doi.org/10.1016/0010-0277(82)90023-3

Keren, G., \& Teigen, K. H. (2001). The probability - outcome correspondence principle: A dispositional view of the interpretation of probability statements. Memory and Cognition, 29, 1010-1021.

Kuipers, B., Moskowitz, A. J., \& Kassirer, J. P. (1988). Critical Decisions under Uncertainty: Representation and Structure. Cognitive Science, 12, 177-210.

Kuperman, V., Stadthagen-Gonzalez, H., \& Brysbaert, M. (2012). Age-of-acquisition ratings for 30,000 English words. Behavior Research Methods, 44, 978-990. doi: 10.3758/s13428$012-0210-4$

Lagnado, D. A., \& Sloman, S. A. (2007). Inside and outside probability judgment. In D. J. Koehler \& N. Harvey (Eds.), (pp. 155-176): Oxford: Blackwell Publishing Ltd.

Løhre, E., \& Teigen, K. H. (2016). There is a $60 \%$ probability, but I am $70 \%$ certain: Communicative consequences of external and internal expressions of uncertainty. Thinking and Reasoning. doi: http://dx.doi.org/10.1080/13546783.2015.1069758

MacLeod, A., \& Pietravalle, S. (2017). Communicating risk: variability of interpreting qualitative terms. EPPO Bulletin, 47, 57-68. doi: 10.1111/epp.12367

Mazur, D. J., \& Hickam, D. H. (1991). Patients' interpretation of probability terms. Journal of General Internal Medicine, 6, 237-240.

Merz, J. F., Druzdzel, M. J., \& Mazur, D. J. (1991). Verbal Expressions of Probability in Informed Consent Litigation. Medical Decision Making, 11, 273-281. doi: http://dx.doi.org/10.1177/0272989X9101100405

Ministry of Defence. (2011). Understanding and intelligence support to joint operations. In M. o. Defence (Ed.): The Development, Concepts and Doctrine Centre. 
Moxey, L. M., \& Sanford, A. J. (1993). Prior expectation and the interpretation of natural language quantifiers. European Journal of Cognitive Psychology, 5, 73-91. doi: http://dx.doi.org/10.1080/09541449308406515

Moxey, L. M., \& Sanford, A. J. (2000). Communicating quantities: A review of psycholinguistic evidence of how expressions determine perspectives. Applied Cognitive Psychology, 14, 237-255. doi: http://dx.doi.org/10.1002/(SICI)1099-0720

Moxey, L. M., Sanford, A. J., \& Dawydiak, E. J. (2001). Denials as controllers of negative quantifier focus. Journal of Memory and Language, 44, 427-442. doi: http://dx.doi.org/doi:10.1006/jmla.2000.2736

Mullet, E., \& Rivet, I. (1991). Comprehension of verbal probability expressions in children and adolescents. Language \& Communication., 11, 217-225. doi: http://dx.doi.org/10.1016/0271-5309(91)90007-I

Neuner-Jehle, S., Senn, O., Wegwarth, O., Rosemann, T., \& Steurer, J. (2011). How do family physicians communicate about cardiovascular risk? Frequencies and determinants of different communication formats. BMC Family Practice 2011, 12:15, 12. doi: http://dx.doi.org/10.1186 /1471-2296-12- 15

Olson, M. J., \& Budescu, D. V. (1997). Patterns of preference for numerical and verbal probabilities. Journal of Behavioral Decision Making, 10, 117-131.

Reagan, R. T., Mosteller, F., \& Youtz, C. (1989). Quantitative meanings of verbal probability expressions. Journal of Applied Psychology, 74, 433-442.

Renooij, S., \& Witteman, C. (1999). Talking probabilities: Communicating probabilistic information with words and numbers. International Journal of Approximate Reasoning, 22, 169-194. 
Robinson, E. J., Pendle, J. E. C., Rowley, M. G., \& Beck, S. R. (2009). Guessing imagined and live chance events: Adults behave like children with live events. British Journal of Psychology, 100, 645-659. doi: http://dx.doi.org/10.1348/000712608X386810

Sirota, M., \& Juanchich, M. (2012). To what extent do politeness expectations shape risk perception? Even numerical probabilities are under the spell! Acta Psychologica, 141, 391-399. doi: http://dx.doi.org/10.1016/j.actpsy.2012.09.004

Teigen, K. H., \& Brun, W. (1995). Yes, but it is uncertain - Direction and communicative intention of verbal probabilistic terms. Acta Psychologica, 88, 233-258. doi: http://dx.doi.org/10.1016/0001-6918(93)E0071-9

Teigen, K. H., \& Brun, W. (2000). Ambiguous Probabilities: When Does p = 0.3 Reflect a Possibility, and When Does It Express a Doubt? Journal of Behavioral Decision Making, $13,345-362$.

Teigen, K. H., \& Brun, W. (2003). Verbal expressions of uncertainty and probability. In D. Hardman \& L. Macchi (Eds.), (pp. 125-145): Chichester, UK: John Wiley \&amp; Sons.

Ülkümen, G., Fox, C. R., \& Malle, B. F. (2016). Two Dimensions of Subjective Uncertainty: Clues from Natural Language. Journal of Experimental Psychology: General, 145, 12801297.

Wallsten, T. S., \& Budescu, D. V. (1995). A review of human linguistic probability processing: General principles and empirical evidence. The Knowledge Engineering Review, 10, 4362. doi: http://dx.doi.org/10.1017/S0269888900007256

Wallsten, T. S., Budescu, D. V., \& Erev, I. (1988). Understanding and using linguistic uncertainties. Acta Psychologica, 68, 39-52. doi: http://dx.doi.org/10.1016/0001$\underline{6918(88) 90044-3}$ 
Wallsten, T. S., Budescu, D. V., Zwick, R., \& Kemp, S. M. (1993). Preferences and reasons for communicating probabilistic information in verbal or numerical terms. Bulletin of the Psychonomic Society, 31, 135-138. doi: 10.3758/BF03334162

Witteman, C. L. M., \& Renooij, S. (2003). Evaluation of a verbal-numerical probability scale. International Journal of Approximate Reasoning, 33, 117-131. doi: http://dx.doi.org/10.1016/S0888-613X(02)00151-2

Witteman, C. L. M., Renooij, S., \& Koele, P. (2007). Medicine in words and numbers: A crosssectional survey comparing probability assessment scales. BMC Medical Informatics and Decision Making, 7, 13-20. doi: http://dx.doi.org/10.1186/1472-6947-7-13

Xu, J.-H., Ye, X.-B., \& Li, S. H. U. (2009). Communication Mode Preference Paradox Among Native Chinese Speakers. The Journal of Social Psychology, 149, 128-129. doi: 10.3200/SOCP.149.1

Young, S., \& Oppenheimer, D. M. (2009). Effect of communication strategy on personal risk perception and treatment adherence intentions. Psychology, Health \&amp; Medicine, 14, 430-442.

Zimmer, A. C. (1983). Verbal versus numerical processing of subjective probabilities. In R. W. Scholz (Ed.), (pp. 159-182): Amsterdam: Elsevier. 
Table appendix - inter-coder reliability across outcomes for Experiments 1 and 2.

$$
\text { Experiment } 1(N=55) \quad \text { Experiment } 2(N=201)
$$

\section{Epistemic}

French village

1

Norwegian fish

1

1

1

Dispositional (animate)

Blood donation

1

1

Bungee jump

0.95

0.99

\section{Dispositional (inanimate)}

Tyre

Migraine

Distributional

Die

1

0.85

Cards

0.96

1 